\title{
Mid-infrared spectroscopic analysis of raw milk to predict the blood nonesterified fatty acid concentrations in dairy cows
}

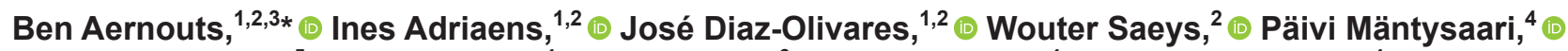 \\ Tuomo Kokkonen, ${ }^{5} \odot$ Terhi Mehtiö, ${ }^{4}{ }^{\oplus}$ Sari Kajava, ${ }^{6} \odot$ Paula Lidauer, ${ }^{4} \odot$ Martin H. Lidauer, ${ }^{4} \odot$ \\ and Matti Pastell ${ }^{3}$ () \\ ${ }^{1}$ KU Leuven, Department of Biosystems, Biosystems Technology Cluster, Campus Geel, Kleinhoefstraat 4, 2440 Geel, Belgium \\ ${ }^{2} \mathrm{KU}$ Leuven, Department of Biosystems, Mechatronics, Biostatistics and Sensors Division, Kasteelpark Arenberg 30, 3001 Leuven, Belgium \\ ${ }^{3}$ Natural Resources Institute of Finland (Luke), Maarintie 6, 02150 Espoo, Finland \\ ${ }^{4}$ Natural Resources Institute of Finland (Luke), Tietotie 4, 31600 Jokioinen, Finland \\ ${ }^{5}$ University of Helsinki, Department of Agricultural Sciences, Koetilantie 5, 00014 Helsinki, Finland \\ ${ }^{6}$ Natural Resources Institute of Finland (Luke), Halolantie 31 A, 71750 Maaninka, Finland
}

\section{ABSTRACT}

In high-yielding dairy cattle, severe postpartum negative energy balance is often associated with metabolic and infectious disorders that negatively affect production, fertility, and welfare. Mobilization of adipose tissue associated with negative energy balance is reflected through an increased level of nonesterified fatty acids (NEFA) in the blood plasma. Earlier, identification of negative energy balance through detection of increased blood plasma NEFA concentration required laborious and stressful blood sampling. More recently, attempts have been made to predict blood NEFA concentration from milk samples. In this study, we aimed to develop and validate a model to predict blood plasma NEFA concentration using the milk mid-infrared (MIR) spectra that are routinely measured in the context of milk recording. To this end, blood plasma and milk samples were collected in wk 2, 3, and 20 postpartum for 192 lactations in 3 herds. The blood plasma samples were taken in the morning, and representative milk samples were collected during the morning and evening milk sessions on the same day. To predict plasma NEFA concentration from the milk MIR spectra, partial least squares regression models were trained on part of the observations from the first herd. The models were then thoroughly validated on all other observations of the first herd and on the observations of the 2 independent herds to explore their robustness and wide applicability. The final model could accurately predict blood plasma NEFA concentrations $<0.6 \mathrm{mmol} / \mathrm{L}$ with a root mean square error of prediction of $<0.143 \mathrm{mmol} / \mathrm{L}$. However, for blood plasma with $>1.2 \mathrm{mmol} / \mathrm{L}$ NEFA, the model

Received November 23, 2019

Accepted February 29, 2020.

*Corresponding author: ben.aernouts@kuleuven.be clearly underestimated the true level. Additionally, we found that morning blood plasma NEFA levels were predicted with significantly higher accuracy using MIR spectra of evening milk samples compared with MIR spectra of morning samples, with root mean square error of prediction values of, respectively, 0.182 and $0.197 \mathrm{mmol} / \mathrm{L}$, and $\mathrm{R}^{2}$ values of 0.613 and 0.502 . These results suggest a time delay between variations in blood plasma NEFA and related milk biomarkers. Based on the MIR spectra of evening milk samples, cows at risk for negative energy status, indicated by detrimental morning blood plasma NEFA levels (>0.6 mmol/L), could be identified with a sensitivity and specificity of, respectively, 0.831 and 0.800 . As this model can be applied to millions of historical and future milk MIR spectra, it opens an opportunity for regular metabolic screening and improved resilience phenotyping.

Key words: milk mid-infrared spectroscopy, blood plasma nonesterified fatty acid concentration, negative energy status, milk biomarker

\section{INTRODUCTION}

The transition from pregnancy to lactation in highyielding dairy cows is typically accompanied by a negative energy balance, in which the energy requirement exceeds the energy input from feed. As severe negative energy balance increases the susceptibility to various health and fertility problems (LeBlanc, 2010; Ospina et al., 2010a), the duration and degree of this imbalance should be limited through preventive actions in combination with individual monitoring and imperative treatment.

To compensate for the energy deficit and maintain high milk production, adipose tissue is mobilized and nonesterified fatty acids (NEFA) are released in the blood. Hence, a blood plasma NEFA concentration $>0.6$ 
$\mathrm{mmol} / \mathrm{L}$ is generally used as an indicator for negative energy balance in dairy cattle (Ospina et al., 2010b). These high concentrations of circulating NEFA have a detrimental effect on oocyte quality and the immune response of dairy cows (Leroy et al., 2005; Scalia et al., 2006). In the liver, part of the NEFA are oxidized completely to deliver energy or incompletely to produce ketone bodies (Adewuyi et al., 2005). Another portion of the NEFA is esterified to triglycerides and either stored in the liver or transported as lipoproteins to, for example, the alveolar epithelial cells of the udder tissue to synthesize milk fat. In this way, fatty acids (FA) and ketone bodies derived from NEFA end up in the produced milk. Previous studies have demonstrated the use of milk biomarkers to monitor negative energy status in individual cows; for example, through the measurement of certain FA (Van Haelst et al., 2008; Jorjong et al., 2014; Dórea et al., 2017), ketone bodies (Enjalbert et al., 2001), citrate, and many more (Bjerre-Harpøth et al., 2012). In contrast to taking blood samples, milk sampling requires less labor and can be done without distressing the animals. Nevertheless, the reference techniques to measure these milk biomarkers are typically labor intensive and costly (Jorjong et al., 2014).

A relatively straightforward and cost-efficient technique for milk analysis is mid-infrared (MIR) spectroscopy. As the covalent bonds of molecules in milk absorb MIR radiation at very specific wavenumbers, the concentrations of these milk components can be derived from MIR absorbance spectra. Typically, multivariate linear models are trained to predict milk constituents from the acquired spectra (De Marchi et al., 2014). This technique has been used for decades and is accepted as the reference for accurate and routine characterization of the main milk components in the context of milk recording (ISO, 2013; ICAR, 2019). Since the commercial introduction of Fourier-transform MIR spectrometers for milk analysis, milk MIR spectra can be obtained with high accuracy and repeatability. This opens opportunities for measuring minor milk components and milk biomarkers such as FA profiles (Rutten et al., 2009; Afseth et al., 2010; Soyeurt et al., 2011), protein composition (Franzoi et al., 2019), minerals (Soyeurt et al., 2009), ketone bodies, and citrate (Grelet et al., 2016).

Recently, Benedet et al. (2019), Grelet et al. (2019), and Luke et al. (2019) developed models to predict blood plasma NEFA concentrations from milk MIR spectra of individual dairy cows. The prediction performance of Grelet's model was poor $\left(\mathrm{R}^{2}=0.39\right)$, whereas Benedet's model performed better $\left(\mathrm{R}^{2}=0.52\right)$. Nevertheless, neither model was validated for a completely independent herd (Benedet et al., 2019; Grelet et al.,
2019). Accordingly, the reported results might be overoptimistic compared with applying the model on the data of a new herd where the cows are managed differently. This was clearly illustrated by Luke et al. (2019); the coefficient of determination $\left(\mathbf{R}^{2}\right)$ of their model decreased from 0.61 for a randomly selected validation set, covering the same herds as those included in the calibration set, to 0.45 for a completely independent herd.

We hypothesized that better prediction performance can be obtained by increasing the number of calibration samples and applying very strict timing in the sampling of blood and milk samples relative to the diurnal pattern and feeding schedule of the cows (Quiroz-Rocha et al., 2010). To test this hypothesis, we collected a large number of samples following a strict protocol for blood and milk sample collection to obtain high-quality data for training the prediction models. Additionally, we investigated whether MIR spectra of morning or evening milk samples resulted in better prediction of NEFA concentrations in the blood samples taken in the morning of that day. Finally, the performance of the prediction models was evaluated extensively on a completely independent validation set.

\section{MATERIALS AND METHODS}

\section{Experimental Setup}

The experimental protocol was approved by the Finnish Animal Experiment Board (ESAVI/5688/04.10.07/2013) and applied on 3 experimental herds in Finland: Luke Jokioinen (herd A), University of Helsinki in Viikki (herd B), and Luke Kuopio (herd C). All cows in these herds that calved for the first time between September 2013 and October 2016 were included in the study, resulting in a total of 143 Nordic Red dairy cows (103 in herd A, 24 in herd B, and 16 in herd C). For 49 of these 143 cows, their second lactation was also included in the study period, resulting in a total of 192 lactations. All cows in herds A and C were housed in a freestall barn, whereas some cows in herd B were kept in tiestalls in early lactation before being moved to a freestall barn. All cows were milked twice a day, with the morning milking between 0630 and $0800 \mathrm{~h}$ and the evening milking between 1600 and $1730 \mathrm{~h}$. The cows in herds A, B, and C were milked in a $2 \times 6$ auto-tandem milking parlor, an automatic milking system, and a $2 \times 8$ herringbone milking parlor, respectively. All cows had ad libitum feeding and were fed grass silage and a concentrate mix. In herds A and $\mathrm{C}$, silage was fed 4 times a day, and concentrate was provided separately using concentrate feeders with 5 
feeding periods per day. In addition, each cow received $0.3 \mathrm{~kg}$ of concentrate during each milking. The cows of herd B kept in tiestalls were fed grass silage and concentrate separately, whereas cows in the freestall barn received a partial mixed ration of grass silage and concentrate and additional concentrate from the milking robot. Further details on the ration and mean intakes of silage and concentrate are provided in Mäntysaari et al. (2019). Cows with clinical signs of ketosis received an oral supplementation of $230 \mathrm{~g}$ of propylene glycol twice a day during the period of the symptoms. Cases of severe ketosis were additionally treated with a single i.m. cortisone injection (dexamethasone $0.04 \mathrm{mg} / \mathrm{kg}$ ).

\section{Data Collection}

For each lactation, blood samples were taken from the coccygeal vein within $1 \mathrm{~h}$ after the morning milking session, on 2 nonconsecutive days each in wk 2 and 3 after calving and once in wk 20. This sampling rate was chosen because cows have an elevated risk of negative energy balance in wk 2 and 3, and thus increased blood NEFA concentrations are expected. At wk 20, on the other hand, cows are beyond the period of maximal energy requirement, typically resulting in low blood NEFA concentrations. Accordingly, both high and low blood NEFA concentrations were included. This approach was needed to train a model that can operate over the entire concentration range. This sampling scheme resulted in a total of 5 blood samples per lactation. Handling of the lactating cows before blood sampling was minimized to reduce its effect on blood plasma NEFA concentrations (Leroy et al., 2011). Blood was collected in 10-mL EDTA tubes and stored in ice until centrifuged at $-4^{\circ} \mathrm{C}$ for $15 \mathrm{~min}$ at $2,000 \times g$. Plasma samples were frozen and stored at $-20^{\circ} \mathrm{C}$ for later analysis of NEFA at the university of Helsinki (Salin et al., 2012). An enzymatic colorimetric acyl-CoA synthetase (ACS)-acyl-CoA oxidase (ACOD) method [NEFA-HR(2) kit, Wako Chemicals GmbH, Neuss, Germany] was used according to the manufacturer's instructions to determine the blood plasma NEFA concentrations, further referred to as "blood NEFA." Intra- and interassay coefficients of variation for blood NEFA determination were 1.61 and $3.53 \%$ for low NEFA concentration $(0.23 \mathrm{mmol} / \mathrm{L})$ and 0.77 and $2.91 \%$ for high NEFA concentration $(1.24 \mathrm{mmol} / \mathrm{L})$.

Representative milk samples $( \pm 30 \mathrm{~mL})$ were collected during the morning and evening milking sessions on the same days as the blood collection, providing a total of 10 milk samples per lactation. The milk samples were stored at $4^{\circ} \mathrm{C}$ using a preservative $( \pm 0.3 \mathrm{mg}$ of bronopol per $\mathrm{mL}$ of milk, Broad Spectrum Microtabs II, D\&F
Table 1. Number of mid-infrared (MIR) transmittance spectra of morning and evening milk samples available for the 3 herds included in this study

\begin{tabular}{lcccc}
\hline & & \multicolumn{3}{c}{ Herd } \\
\cline { 3 - 5 } Milk samples & All herds & $\mathrm{A}$ & $\mathrm{B}$ & $\mathrm{C}$ \\
\hline Morning & 808 & 640 & 121 & 47 \\
Evening & 777 & 646 & 118 & 13 \\
\hline
\end{tabular}

Control Systems Inc., Dublin, CA). The MIR analyses (MilkoScan FT6000 spectrometer, Foss, Hillerød, Denmark) were carried out by the Valio Ltd. milk laboratory (Seinäjoki, Finland) according to ISO 9622:2013 (ISO, 2013). The MIR spectrum of each milk sample consisted of 1,060 values, representing infrared light transmittance through $50 \mu \mathrm{m}$ of sample between wavenumbers $5,010.2$ and $925.7 \mathrm{~cm}^{-1}$ with a resolution of 4 $\mathrm{cm}^{-1}$. The MIR spectra were standardized following the procedure developed by Grelet et al. (2015). Because of data storage problems, the MIR spectra of 152 morning milk samples and 183 evening milk samples were lost. The resulting final data set therefore included 808 and 777 MIR spectra for morning and evening milk samples, respectively (Table 1).

\section{Prediction of Blood NEFA Concentrations from Milk MIR Spectra}

The milk MIR spectra, blood NEFA concentrations, and respective cow identifier numbers and sampling dates and times were imported into $\mathrm{R}$ (version 3.4.3; $\mathrm{R}$ Core Team, 2017). The cow identifier numbers and sampling dates and times were solely used to relate blood NEFA concentrations to the correct milk MIR spectra. The prediction model thus only used the milk MIR spectra as input to predict the blood NEFA concentration. All observations were considered by the prediction model to be totally independent. Only the spectral regions from 2,977 to $2,768 \mathrm{~cm}^{-1}, 1,800$ to $1,684 \mathrm{~cm}^{-1}$ and 1,607 to $926 \mathrm{~cm}^{-1}$ were used in the analysis. Moreover, the signal-to-noise ratio in the spectral regions between 3,660 and 2,977 $\mathrm{cm}^{-1}$ and between 1,684 and 1,607 $\mathrm{cm}^{-1}$ was considered too low because of substantial MIR absorption by water molecules. The spectral regions above $3,660 \mathrm{~cm}^{-1}$ and between 2,768 and $1,800 \mathrm{~cm}^{-1}$ were deleted because they do not contain significant spectral information on relevant milk components (Aernouts et al., 2011; Grelet et al., 2019). A principal component analysis (PCA) with a maximum of 20 principal components was used to identify potential outlier spectra. For each of the 20 PCA models, the root mean square error of cross-validation (RMSECV) was calculated 
from the difference between the actual spectra and those predicted (in the cross-validation procedure) with the "missing data" approach (Bro et al., 2008). Accordingly, the PCA model with the minimum RMSECV was selected and the Hotelling $T^{2}$ statistic versus $Q$ residuals (influence plot) was studied. When both the $Q$ residuals and the Hotelling $T^{2}$ statistic were above their $99 \%$ confidence limits, the spectrum was removed from the analysis (Bro and Smilde, 2014).

As blood samples were only taken once per cow per sampling day, whereas 2 milk samples were collected for the morning and evening milking sessions of that day and cow, the number of blood NEFA analyses was half the number of milk MIR spectra. Accordingly, the same blood NEFA concentration was assigned to both the morning and the evening milk MIR spectrum of the respective cow and day. The combination of a morning milk MIR spectrum together with its respective blood NEFA concentration is further referred to as a "morning observation," whereas the combination of an evening milk MIR spectrum together with its respective blood NEFA concentration is further referred to as an "evening observation." The morning and evening observations of the same cow and day thus had the same blood NEFA concentration but different milk MIR spectra. Next, about $60 \%$ of the morning and evening observations of herd A were allocated to the calibration set, and the remaining $40 \%$ of the observations of herd $\mathrm{A}$ and all observations of herds $\mathrm{B}$ and $\mathrm{C}$ were assigned to the validation set (Figure 1, step 1). Moreover, the observations of herd A were split 60:40 by applying the duplex selection method after ordering them on their blood NEFA concentration (Snee, 1977). This procedure ensured that both sets had similar descriptive statistics. Observations for the same cow were treated as a block with all of them either in the calibration or validation set to prevent overoptimistic validation results in case of modeling cow-specific effects (Kemps et al., 2010). This data structure allowed us to evaluate the envisaged prediction model on independent observations of the herd used for calibration (herd A), as well as 2 independent herds (B and $\mathrm{C}$ ). Comparing the model performances for the 3 herds will provide deep insights into the robustness and applicability of the model.

The spectral preprocessing of the MIR spectra was a combination of (1) a logarithmic spectral transformation (Beer, 1852) or not; (2) a baseline correction, detrending, standard normal variates weighting, or multiplicative scatter correction (Geladi et al., 1985; Barnes et al., 1989; Ruckstuhl et al., 2001) or none of those; (3) a first- or second-order Savitzky-Golay derivative (Savitzky and Golay, 1964) with a secondorder polynomial filter and 10 different spectral window lengths (3 to 21 spectral wavenumbers in steps of 2) or no derivative; and (4) mean-centering. This resulted in 210 different combinations, as presented in Figure 1 (step 2) and described in detail in Aernouts et al. (2011). For each of these 210 combinations, a partial least squares regression (PLSR) model with up to 20 latent variables was built to predict blood NEFA concentrations, further referred to as "predicted blood NEFA," from the preprocessed MIR spectra (Martens and Næs, 1987). A group-wise cross-validation with 20 groups, each containing spectra of 3 to 4 cows, was performed on the observations of the calibration set to obtain the prediction error for each observation and calculate the RMSECV from them. In each crossvalidation iteration, all observations of a cow were either in or out of the training set to prevent modeling cow-specific effects or relations (Kemps et al., 2010). We selected the smallest number of latent variables for which the PLSR model was not significantly worse than

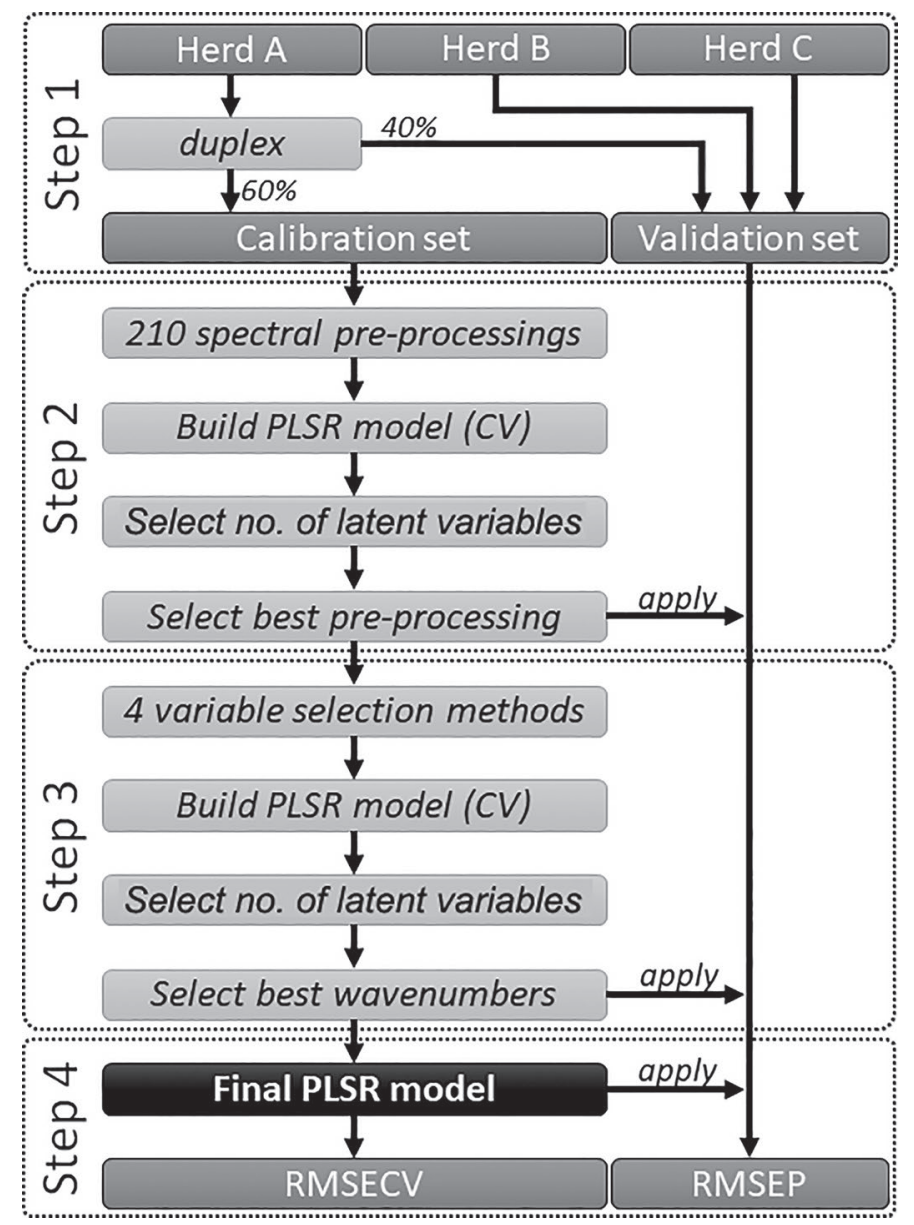

Figure 1. Schematic overview of the methodology to build a partial least squares (PLSR) model to predict the blood plasma nonesterified fatty acid concentration from milk mid-infrared spectra. CV $=$ crossvalidation, RMSECV = root mean square error of cross-validation, $\mathrm{RMSEP}=$ root mean square error of prediction. 
the respective model with the number of latent variables resulting in the lowest RMSECV. The statistical comparison in this procedure was based on a one-sided paired $T$-test $(\alpha=0.05)$ applied on the absolute residuals of the cross-validated observations (Cederkvist et al., 2005). A similar approach was followed to select the best spectral preprocessing combination. Moreover, the PLSR models resulting from the 210 combinations were ranked by increasing RMSECV, and that with the smallest number of latent variables and not significantly worse than the model with the lowest RMSECV was selected. Again, a one-sided paired $T$-test $(\alpha=$ $0.05)$ on the absolute residuals of the cross-validated observations was used to compare the models statistically (Cederkvist et al., 2005; Aernouts et al., 2011).

The selected preprocessing combination was applied on the MIR spectra to be used as an input for 4 different variable selection methods (Figure 1, step 3): variable importance in projection, jack-knife, reversed interval PLSR, and forward interval PLSR (Norgaard et al., 2000; Westad and Martens, 2000; Chong and Jun, 2005). Each of these 4 methods resulted in a set of most relevant wavenumbers for which a PLSR model with an optimal number of latent variables was built, as described earlier. The performances of these 4 PLSR models were compared mutually and with the model that used all wavenumbers. Finally, the set of wavenumbers related to the most parsimonious model whose prediction performance was not significantly worse (one-sided paired $T$-test, $\alpha=0.05$ ) than that of the model with the lowest RMSECV was selected.

The final prediction model (Figure 1, step 4), together with the selected combination of spectral preprocessing techniques and the selected set of wavenumbers, was used to predict the NEFA concentrations of the observations in the validation set. Accordingly, an error or residual could be calculated for each observation of the validation set. Based on these residuals, the root mean square error of prediction (RMSEP), further referred to as the "prediction error," was calculated for the entire validation set. Because this validation set is very diverse, containing morning and evening observations from 3 different herds with blood NEFA concentrations ranging from very low to very high, the RMSEP was also calculated for different subsets of the validation set, allowing for a better understanding of the prediction performance of the model under different situations. These subsets were defined based on a combination of the following features:

- Milking time: only morning observations, only evening observations, or both morning and evening observations;
- Herd: observations from herd A, herd B, herd C, or for the 3 herds together;

- NEFA range: observations with blood NEFA concentrations in the low $(0-0.6 \mathrm{mmol} / \mathrm{L})$, middle $(0.6-1.2 \mathrm{mmol} / \mathrm{L})$, high $(1.2-2.0 \mathrm{mmol} / \mathrm{L})$, or complete range $(0-2 \mathrm{mmol} / \mathrm{L})$. These ranges were defined as such because $0.6 \mathrm{mmol} / \mathrm{L}$ is generally considered the critical threshold (Ospina et al., 2010b) and because blood NEFA concentration was always underestimated for true concentrations above $1.2 \mathrm{mmol} / \mathrm{L}$.

The procedure described in Figure 1 was initially followed to develop and validate a PLSR model that predicts blood NEFA independent of the moment of milk sampling by training it on all the observations - both morning and evening - of the calibration set. This model is further referred to as the "full model." To evaluate the effect of restricting the calibration set to only morning or evening observations, 2 new models were trained following the same procedure as elaborated in Figure 1, but with only the morning or the evening observations of the calibration set to train the respective PLSR models. These models are further referred to as the "morning model" and the "evening model." All 3 models (full, morning, and evening) were validated on the same observations - both morning and evening - of the validation set to allow for an objective comparison of prediction performance.

The prediction performance of the 3 models was compared by applying a repeated-measures ANOVA on the absolute residuals for all the observations of the validation set. Moreover, "model" was treated as a fixed effect, whereas "sample" was specified as a random effect in the 2-way ANOVA (Cederkvist et al., 2005). When the ANOVA test pointed out a significant effect $(\alpha=0.05)$ of the model, then the performance of the 3 models was compared bilaterally using a Tukey honestly significant difference (HSD) multiple comparison $(\alpha=0.05)$. The 3 models were compared for all observations in the validation set, as well as the observations in the different subsets of the validation set. The model (full, morning, or evening) that was not significantly different from the best model for most of the subsets of the validation set was identified as the most robust. This model was further evaluated on its ability to identify detrimental blood NEFA concentrations (see next section). Finally, a 4-way ANOVA, with model, milking time, herd, and NEFA range, and all possible interactions as fixed factors, was applied on the absolute residuals for the observations of the entire validation set and subsets of the validation set. This analysis was not paired, so the samples could not be taken as a random factor. If one 
of the interactions was significant $(\alpha=0.05)$, then all possible combinations of the factors involved in these interactions were compared bilaterally using the Tukey HSD multiple comparisons. In the absence of significant interaction for a factor, the effect of the factors could be interpreted separately. Moreover, if this factor had a significant $(\alpha=0.05)$ influence on performance, then the different levels within this factor were compared bilaterally with the Tukey HSD multiple comparisons.

\section{Identifying Detrimental Blood NEFA Concentrations from Milk MIR Spectra}

To evaluate whether the predicted blood NEFA concentrations could be used to identify detrimental blood NEFA levels $(>0.6 \mathrm{mmol} / \mathrm{L})$, receiver operating characteristic (ROC) analyses were performed (Ospina et al., 2010b; Jorjong et al., 2014; Dórea et al., 2017). The ROC curves plot the true positive rate or sensitivity versus the true negative rate (1 - specificity) for different thresholds applied on the predicted blood NEFA concentration. Only the most robust model, the one that performed best according to the procedure described in the previous section, was subjected to this ROC analysis. A separate analysis was done for the morning and evening observations of the validation set. The R package pROC (version 1.13.0; Robin et al., 2011) was used to calculate the ROC curves, to apply binormal smoothing to the ROC curves, to calculate the $95 \%$ confidence intervals (CI) of sensitivities, specificities, and area under the curve (AUC) of the smoothed ROC curves, and to statistically compare the smoothed ROC curves. The CI were calculated with 100,000 bootstrap replicates to obtain a fair estimate of the second significant digit (Fawcett, 2006). Statistical 2-sided pairwise comparisons $(\alpha=0.05)$ between ROC were done based on the AUC and based on the sensitivities at given specificities from 0 to 1 in steps of 0.01 , both using the bootstrap method with 100,000 replicates.

\section{RESULTS}

\section{Data Exploration}

Table 2 gives an overview of the frequency of observations for which the blood NEFA levels were $>0.6$ $\mathrm{mmol} / \mathrm{L}$ in function of the herd $(\mathrm{A}, \mathrm{B}$, or $\mathrm{C})$ and the lactation stage $(2,3$, or $20 \mathrm{wk})$ of the respective cows. These data clearly show that the prevalence of detrimental blood NEFA concentrations decreased with lactation stage and was $0 \%$ at wk 20 in lactation. For wk 2 in lactation, the highest prevalence (41.0\%) was observed for herd A; for wk 3 in lactation, herd $\mathrm{C}$ had the highest prevalence (23.3\%). Herd B had the lowest prevalence independent of the lactation stage.

The MIR transmittance spectra of the 1,585 milk samples included in this study are presented in the top part of Figure 2 as the black solid and dotted lines. The Hotelling's $T^{2}$ and $Q$ statistics of the PCA model with 7 selected principal components $(99.2 \%$ of spectral variability) and the scores for the first 2 principal components of that model were far beyond the $99 \%$ confidence limits for the spectra of sample \#1445 (herd $\mathrm{B}$, evening milking) and sample \#1546 (herd C, morning milking), as shown in Appendix Figure A1. Also, the raw transmittance spectra of these 2 outliers, illustrated with black dotted lines in the top part of Figure 2 , are clearly different from the other 1,583 spectra, whereas the corresponding blood NEFA concentrations are not outliers. This suggests that these 2 samples have erroneous spectral measurements; they were therefore removed from the data set.

The reliability, accuracy, and robustness of spectroscopic calibrations are restricted to the range of the constituent of interest and the variation in measurement conditions considered during calibration (Williams and Norris, 2001). The descriptive statistics of the blood NEFA concentrations linked to different subsets of milk MIR transmittance spectra are presented in Table 3. The entire calibration and validation sets contained, respectively, 790 and 793 observations and had very similar means, standard deviation, and ranges for blood NEFA. Table 3 also illustrates the larger variability and range of blood NEFA levels in herd A compared with herd B and C. Likely, this is the result of the higher number of blood samples collected and analyzed ( $\mathrm{n}$ $=658)$ and cows being monitored $(\mathrm{n}=103)$ in herd A. Additionally, this might be caused by differences in genetics and management between the herds. The descriptive statistics for morning and evening samples in a same herd(s) were similar but not identical. This is because, for some blood samples, only the morning or evening milk MIR spectra were collected, not both (Table 1).

Table 2. Frequency (\%) of observations for which the blood nonesterified fatty acid levels were $>0.6 \mathrm{mmol} / \mathrm{L}$ by lactation stage (wk) and herd

\begin{tabular}{lccc}
\hline Lactation stage & Herd A & Herd B & Herd C \\
\hline $2 \mathrm{wk}$ & 41.0 & 20.4 & 28.0 \\
$3 \mathrm{wk}$ & 21.2 & 8.5 & 23.3 \\
$20 \mathrm{wk}$ & 0 & 0 & 0 \\
\hline
\end{tabular}




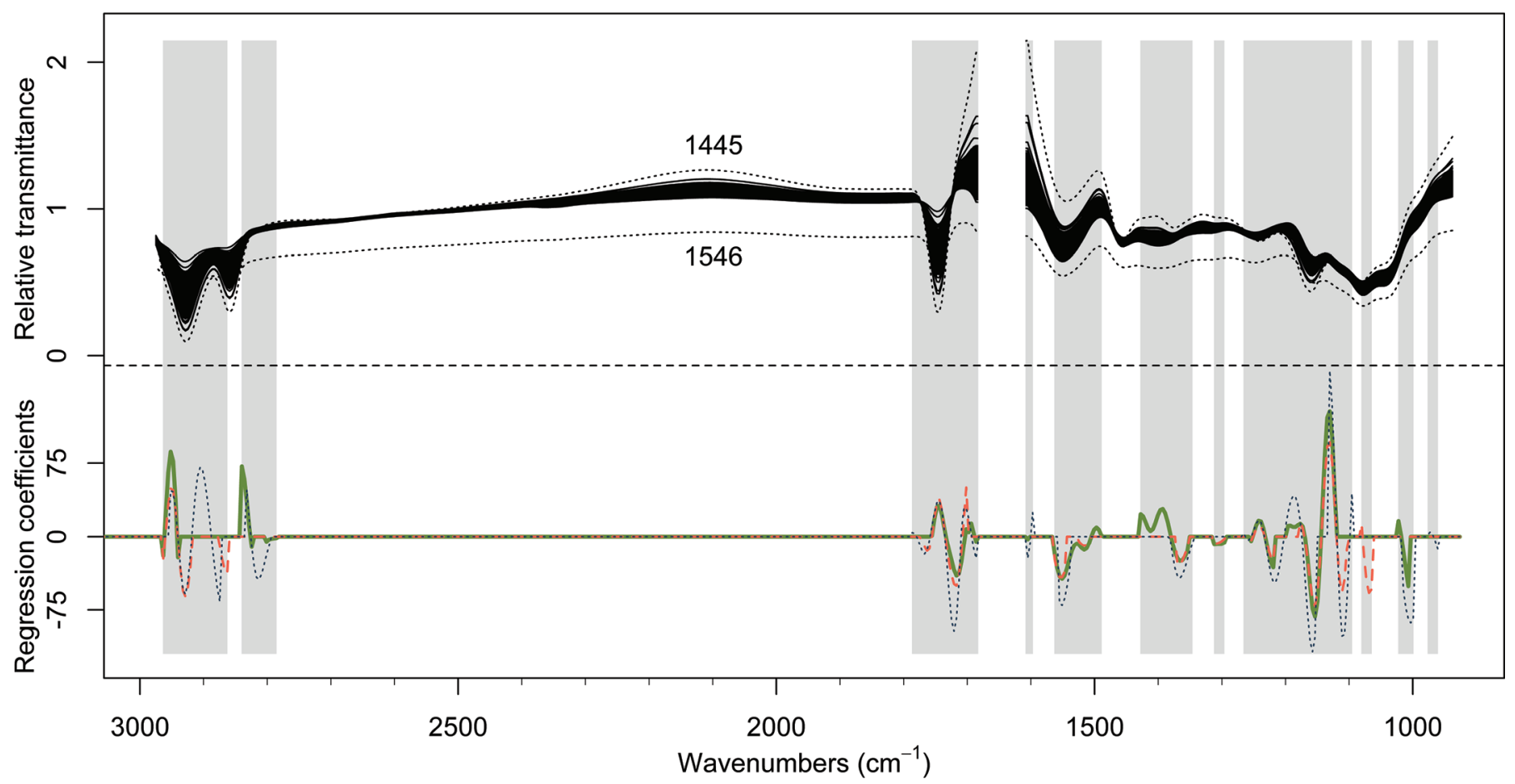

Figure 2. Top: relative mid-infrared (MIR) transmittance spectra of the milk samples (top). The dotted black lines (with sample number) indicate 2 potential outlier spectra. The gray regions indicate the wavenumbers included in at least 1 of the 3 final partial least squares regression (PLSR) models (full, morning, or evening) to predict the blood plasma nonesterified fatty acid concentration after applying a variable selection technique. Bottom: regression coefficients for the 3 PLSR models constructed using a calibration set with MIR spectra of respectively (1) morning and evening milk samples (full model, green solid), (2) only morning milk samples (morning model, red dashed), and (3) only evening milk samples (evening model, blue dotted) of herd A.

\section{Calibration on MIR Spectra of Morning and Evening Milk Samples (Full Model)}

The 790 morning and evening observations of the calibration set were used to build a PLSR model (full model) that related blood NEFA concentrations to MIR transmittance spectra. The best performance was obtained when the MIR transmittance spectra were preprocessed using a second-order Savitzky-Golay derivative with a window length of 7 wavenumber variables, followed by mean-centering. After this preprocessing step, the reversed interval PLSR method selected 117 wavenumbers that were most informative and resulted in the best model. The most informative regions of the MIR spectra are indicated as gray regions in Figure 2.

Figure 3a shows RMSECV and RMSEP as a function of the number of latent variables included in the full PLSR model after applying the best preprocessing and selecting the best wavenumbers. Separate RMSEP are provided for the morning and evening observations of the validation set, indicated by $\mathrm{RMSEP}_{\mathrm{M}}$ and $\mathrm{RMSEP}_{\mathrm{E}}$, respectively. The PLSR model with 6 latent variables,

Table 3. Descriptive statistics of the blood plasma nonesterified fatty acid (NEFA) concentrations (mmol/L) linked to different subsets of milk mid-infrared transmittance spectra

\begin{tabular}{|c|c|c|c|c|c|c|c|c|c|c|c|c|}
\hline \multirow{2}{*}{$\begin{array}{l}\text { Blood } \\
\text { NEFA }\end{array}$} & \multicolumn{3}{|c|}{ Calibration set (herd A) } & \multicolumn{9}{|c|}{ Validation set (herds A, B, and C) } \\
\hline & Overall & Morning & Evening & Overall & All herds & Herd A & Herd B & Herd C & All herds & Herd A & Herd B & Herd $\mathrm{C}$ \\
\hline Number & 790 & 395 & 395 & 793 & 412 & 245 & 121 & 46 & 381 & 251 & 117 & 13 \\
\hline Mean & 0.436 & 0.436 & 0.435 & 0.445 & 0.442 & 0.477 & 0.372 & 0.444 & 0.448 & 0.479 & 0.372 & 0.530 \\
\hline SD & 0.296 & 0.296 & 0.296 & 0.286 & 0.280 & 0.316 & 0.187 & 0.246 & 0.292 & 0.324 & 0.188 & 0.298 \\
\hline
\end{tabular}


a)

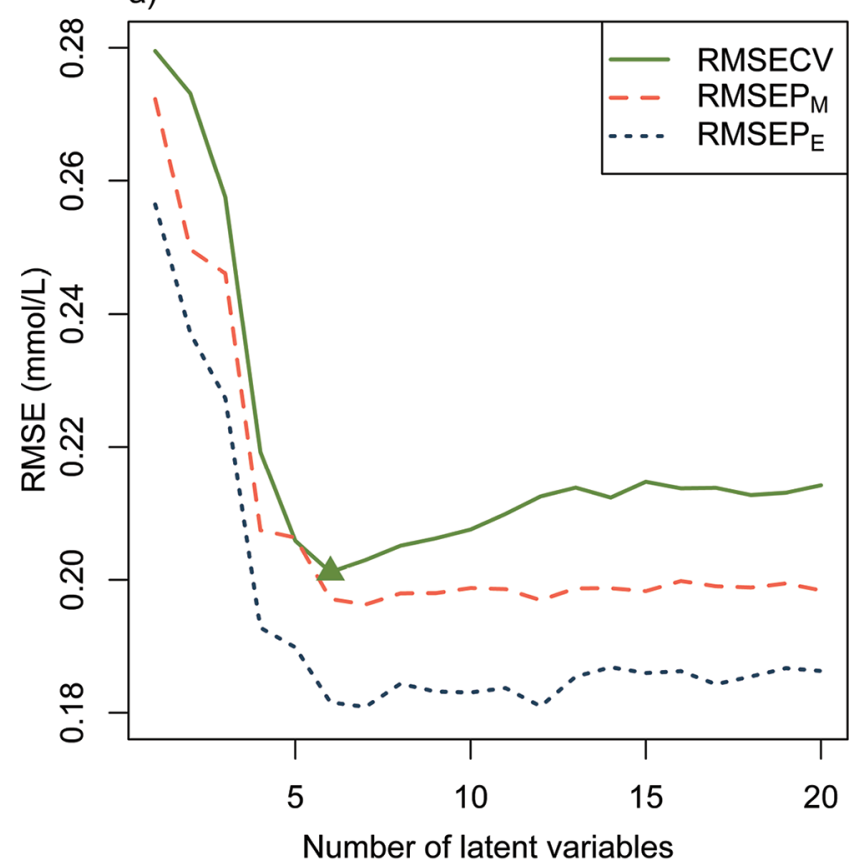

b)

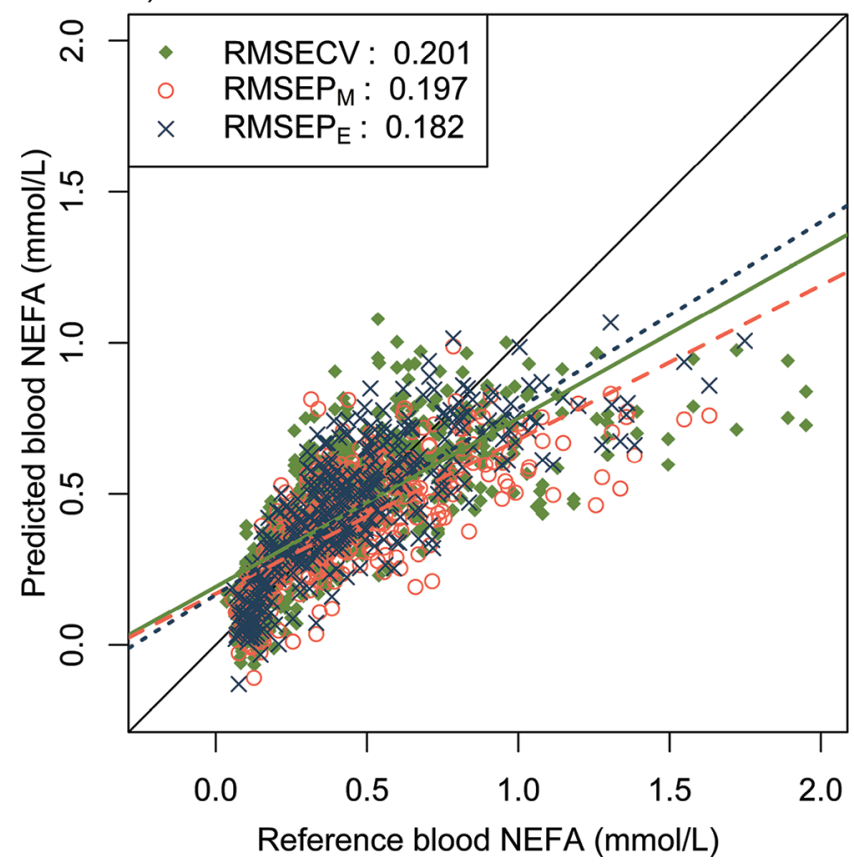

Figure 3. The results of the partial least squares regression (PLSR) model trained on a calibration set of mid-infrared transmittance spectra of milk samples collected during morning and evening milking sessions (full model) on herd A to predict nonesterified fatty acid (NEFA) concentrations in plasma of the respective cows for which blood was sampled in the morning. (a) Root mean squared error (RMSE) for the calibration set in cross-validation $(\mathrm{CV})$ and the morning $\left(\mathrm{P}_{\mathrm{M}}\right)$ and evening $\left(\mathrm{P}_{\mathrm{E}}\right)$ observations of the validation set (all 3 herds), in relation to the number of latent variables of the PLSR model. The green triangle indicates the number of selected latent variables $(n=6)$ for the final PLSR model. (b) Scatterplot of predicted versus measured for the calibration set (herd A) in cross-validation (CV) and the morning $\left(\mathrm{P}_{\mathrm{M}}\right)$ and evening $\left(\mathrm{P}_{\mathrm{E}}\right)$ observations of the validation set (3 herds). indicated with the green triangle, was finally selected. This model complexity resulted in nearly the minimum $\mathrm{RMSEP}_{\mathrm{M}}$ and $\mathrm{RMSEP}_{\mathrm{E}}$, confirming the correct choice of number of latent variables based on the cross-validation and illustrating the robustness of the full model. Figure 3a shows that the RMSEP $\mathrm{E}_{\mathrm{E}}$ was smaller than the $\mathrm{RMSEP}_{\mathrm{M}}$ and that the latter was smaller than the RMSECV.

The regression coefficients for the full model with 6 latent variables are shown as a green solid line in the bottom part of Figure 2. The regression coefficients follow a relatively smooth curve as a function of the wavenumbers, which indicates that the PLSR model was not overfitting the calibration data. High absolute values for the regression coefficients were obtained around $2,950 \mathrm{~cm}^{-1}, 1,750 \mathrm{~cm}^{-1}$ and $1,150-990 \mathrm{~cm}^{-1}$, corresponding to important fat absorption bands: fat $\mathrm{B}$, fat $\mathrm{A}$, and $\mathrm{C}-\mathrm{O}$ stretch vibrations, respectively (Afseth et al., 2010). As the PLSR model uses the second derivative of the MIR spectra, some of the peaks in the regression coefficients are located at the flanks rather than the center of typical absorption peaks.

Figure $3 \mathrm{~b}$ presents the predicted versus measured scatterplot for the full model with 6 latent variables. This figure illustrates that the prediction error of the full model varied a lot with predicted blood NEFA concentration (y-axis), both for the cross-validated observations of the calibration set and for the morning and evening observations of the independent validation set. Additionally, blood NEFA concentration was generally overestimated for true values (x-axis) between 0.2 and $0.55 \mathrm{mmol} / \mathrm{L}$ but always underestimated for true concentrations $>1.2 \mathrm{mmol} / \mathrm{L}$. The latter could explain why the $\mathrm{RMSEP}_{\mathrm{M}}$ and $\mathrm{RMSEP}_{\mathrm{E}}$ were lower than the RMSECV, because the validation set contained fewer observations with a very high blood NEFA concentration (Table 3). In Figure 3b, the predictions based on the evening observations of the validation set (blue crosses) were closer to the identity line than those based on the morning observations of the same set (red circles). This is why the RMSEP $\mathrm{E}_{\mathrm{E}}$ values were smaller than the RMSEP $\mathrm{P}_{\mathrm{M}}$ values in Figure $3 \mathrm{a}$ and it suggests that the blood NEFA concentration in the morning can be predicted more accurately using MIR spectra of milk samples taken in the evening of that day rather than morning milk samples.

The prediction errors of the full model for different subsets of the validation set are summarized in Table 4 . The heteroscedastic prediction error of the full model is clearly shown by the increasing RMSEP with increasing blood NEFA range (different horizontal sections of Table 4). Moreover, for the observations in the low blood NEFA range (0-0.6 mmol/L), the RMSEP values of the full model were all between 0.062 and 0.143 
Table 4. Root mean squared error of prediction (RMSEP) for nonesterified fatty acid (NEFA) concentrations in blood plasma by partial least squares regression models

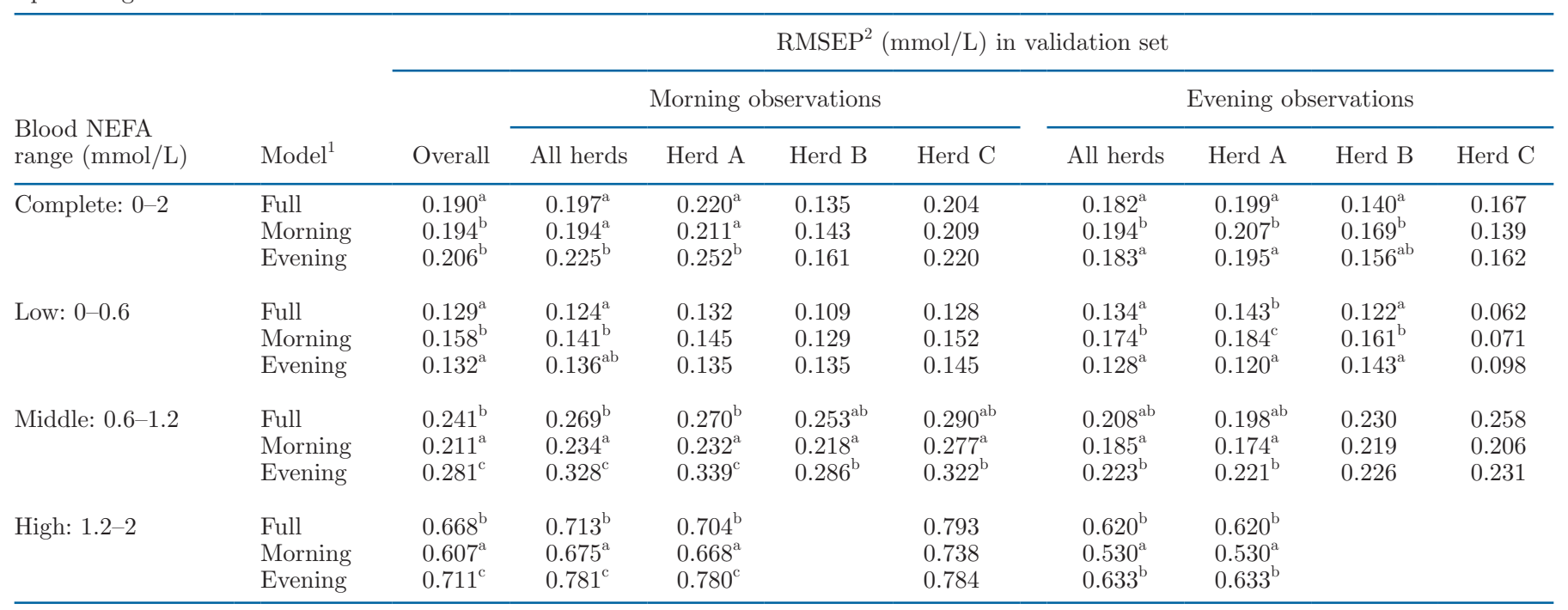

${ }^{\mathrm{a}-\mathrm{c}}$ Within each column and blood NEFA range, RMSEP values with different superscripts indicate significant $(\alpha=0.05)$ differences between the models according to Tukey honestly significant difference (HSD) multiple comparison, with the letter lower in the alphabetical order (from a to c) indicating the better model.

${ }^{1}$ Full model: trained on morning and evening observations; morning model: only morning observations; evening model: only evening observations of the calibration set.

${ }^{2}$ The RMSEP values are provided for different subsets (blood NEFA range, milking time, and herd) of the validation set.

mmol/L, whereas for the middle blood NEFA range (0.6-1.2 $\mathrm{mmol} / \mathrm{L})$, the RMSEP values varied between 0.198 and $0.290 \mathrm{mmol} / \mathrm{L}$. For the high blood NEFA range $(1.2-2 \mathrm{mmol} / \mathrm{L})$, the RMSEP values of the full model were between 0.620 and $0.793 \mathrm{mmol} / \mathrm{L}$. Within the low, middle, and high blood NEFA ranges, the RMSEP values did not differ much between herds, illustrating that the model can be used for new herds as well (e.g., herds B and C). Compared with the morning observations of the validation set, the RMSEP values for evening observations were, in most cases, slightly higher for the low blood NEFA range and clearly lower for the complete, middle, and high blood NEFA ranges. The observations based on the RMSEP values described here were similar to those based on the RMSECV values obtained from the cross-validation of the calibration samples (results not shown), confirming the robustness of the full model.

\section{Calibration on MIR Spectra of Morning or Evening Milk Samples}

The PLSR model trained on the morning observations of the calibration set (morning model) as well as on the evening observations (evening model) provided the best results after applying a second-order SavitzkyGolay derivative on the MIR transmittance spectra, followed by mean-centering. The optimal window length for the derivative was 7 and 13 wavenumber variables, respectively. For both models, reversed interval PLSR proved to be the best variable selection method, resulting in 92 and 126 retained wavenumbers, respectively (gray regions in Figure 2). Finally, 3 and 5 latent variables were selected for the morning and evening PLSR models, respectively. The regression coefficients for the final morning and evening model are presented as, respectively, the red dashed and blue dotted lines in the bottom part of Figure 2. Both models had high absolute values for the regression coefficients in the regions near important MIR fat absorption bands, similar to the regression coefficients of the full model.

The prediction errors of the morning and evening model for different subsets of the validation set are also provided in Table 4, just below the respective RMSEP values of the full model. The prediction performance of the 3 models (full, morning, and evening) was compared for each subset of the validation set. Within each column (herd $\times$ milking time) and a specified blood NEFA range (complete, low, middle, or high), RMSEP values with different superscripts indicate significant $(\alpha$ $=0.05$ ) differences between the 3 models. Most subsets of herd $\mathrm{C}$ and some subsets involving herd B showed no significant difference between the models. For those subsets, we found that the statistical tests lacked power $(\beta>0.4)$ because the number of samples was too small. Therefore, further discussion of model comparisons is 
based only on tests with sufficient power $(\beta<0.2)$, which all indicated a statistical effect of the model. The "Overall" column of Table 4 presents the RMSEP values for all observations of the validation set in each of the 4 specified blood NEFA ranges.

In the complete range, the full model performed significantly better than the morning or evening model, whereas there was no significant difference between the morning and evening models. The full and evening models were not significantly different for observations of the validation set in the low blood NEFA range, but both were significantly better than the morning model. In contrast, for observations in the middle and high NEFA ranges, the morning model was significantly better than the full model, which in turn was better than the evening model.

For the low, middle, and high blood NEFA ranges, the same trends were reflected in the different subsets of the validation set, where the observations are split per herd and milking time (Table 4). For the complete blood NEFA range, the full model was significantly better than the evening model for all validation subsets with only morning observations, whereas it was significantly better than the morning model for all the subsets with evening observations. Additionally, blood NEFA concentrations predicted with the morning model were, on average, $0.042 \mathrm{mmol} / \mathrm{L}$ higher than predictions by the full model applied on the same milk MIR spectra, whereas the evening model resulted in blood NEFA predictions that were, on average, 0.048 $\mathrm{mmol} / \mathrm{L}$ lower than predictions by the full model. Given the fact that low blood NEFA concentrations were generally overestimated by the models, and high blood NEFA concentrations were underestimated (Figure 3b), the morning model resulted in lower predictions in the high NEFA range, and the evening model resulted in lower prediction errors for the low NEFA range. This was clearly reflected by the models' RMSEP values for the different blood NEFA ranges. Because the models rely mainly on absorption by fat-related covalent bonds (Figure 2), the offset between the models probably results from the difference in average fat content between morning milk samples (4.3\%) and evening milk samples (5.1\%) involved in the training set. Taking all this into account, we concluded that the full model was the most robust of the 3 models and it was therefore further explored in the ROC analysis.

Apart from the comparisons between the full, morning, and evening models for each of the subsets of the validation set, a single 4-way ANOVA was performed on the residuals of the observations in these different subsets. Because all but one of the 2-way interactions between the ANOVA factors were significant, the ef- fect of the individual factors could not be interpreted independently of the other factors involved in the interaction(s). Accordingly, all combinations of factors involved in these interactions were compared bilaterally using a Tukey HSD multiple comparison. This analysis mainly indicated that the prediction errors for the middle and high blood NEFA ranges were significantly higher than those for the complete and low ranges, but that the absolute levels of these errors depended on the model, the herd, and the milking time.

Table 4 can also be used to study the differences in prediction error when 1 of the 3 models is applied to different herds or on either morning or evening observations. The RMSEP values for the 3 herds, except for the evening observations of herd $\mathrm{C}$, were very close for the same blood NEFA range for either morning or evening observations. This illustrates that the models can easily be applied in new herds. The RMSEP values for the subsets of evening observations in herd $\mathrm{C}$ should be interpreted with caution because each is based on a small number of observations $(\mathrm{n} \leq 13$, Table 1$)$. Comparing the prediction errors between morning and evening observations for the respective subsets shows that the prediction errors for the morning observations were generally higher, especially for blood NEFA concentrations $>0.6 \mathrm{mmol} / \mathrm{L}$ (middle and high ranges). For the full model, a one-sided paired $T$-test applied on all observations of the validation set showed that the blood NEFA predictions were more accurate $(P=$ 0.009) if the model was applied on evening milk MIR spectra. This confirmed that a blood NEFA concentration in the morning is predicted more accurately from milk MIR spectra taken during the evening milk session of the same day.

The observations based on the RMSEP values described in this section were similar to those based on the RMSECV values obtained from the cross-validation of the calibration samples (results not shown), confirming the robustness of the models and the validity of this analysis.

\section{ROC Analysis of the Full Model}

The smoothed ROC curves for the identification of detrimental blood NEFA concentrations based on the predictions of the full model are shown in Figure 4. Separate smoothed ROC curves are provided for morning (red) and evening observations (blue) of the validation set. The AUC of the smoothed ROC curves for the morning and evening observations are $0.860(95 \%$ CI: $0.815-0.901)$ and 0.898 (95\% CI: $0.860-0.930)$, respectively. Accordingly, the AUC for the morning observations was significantly lower $(P<0.001)$ that that 
for the evening observations. Moreover, compared with evening observations, the sensitivities for the morning observations were significantly lower in the range of specificities from 0.48 to 0.97 . The average sensitivities were 0.752 and 0.573 for the morning observations and 0.831 and 0.690 for the evening observations (Figure 4) at specificities of 0.8 and 0.9 , respectively. Thus, cows with a detrimental blood NEFA concentration, as determined from their morning blood samples, can be detected more accurately using the MIR spectra of their milk collected during the evening milking session of that day. Moreover, the full model can correctly identify 83 out of 100 cows with detrimental blood NEFA concentrations, while 20 out of 100 healthy cows will be wrongly classified as being at risk. Appendix Table A1 provides the mean values of the sensitivities and the $95 \%$ CI of the sensitivities and specificities at given specificities from 0.7 to 0.95 (in steps of 0.05 ) for the morning and evening observations of the validation set.

\section{DISCUSSION}

\section{Morning and Evening Milk Samples}

The validation of the PLSR models clearly indicated that, compared with morning milk, the MIR spectra of evening milk support more accurate predictions of the NEFA levels in blood collected in the morning of that day (Table 4, Figure 3, and Figure 4). Several studies have shown that blood NEFA follows a diurnal pattern, with elevated levels from about 0600 to $1000 \mathrm{~h}$, associated with reduced energy intake during the night (Blum et al., 2000; Meier et al., 2010; Quiroz-Rocha et al., 2010). For this reason, extra attention was paid to consistent timing of the blood and milk sampling. As the morning milking session was between 0630 and 0800 $h$, most of the period of expected elevated blood NEFA was after the morning milking session, thus mainly overlapping with the period in which the evening milk was produced. This likely introduced a time delay between the moment of elevated blood NEFA level and the moment at which a change in the concentration of related milk biomarkers could be noticed, which was further delayed by metabolic processes in the liver that transfer NEFA into milk precursors and constituents (e.g., lipoproteins and ketone bodies). This delay would explain why the blood NEFA concentrations were predicted more accurately from MIR spectra of evening milk than of morning milk because the blood samples were taken within $1 \mathrm{~h}$ after the morning milking session, in the time window of expected elevated blood NEFA levels. This hypothesis is also supported by the slightly higher NEFA levels predicted based on MIR

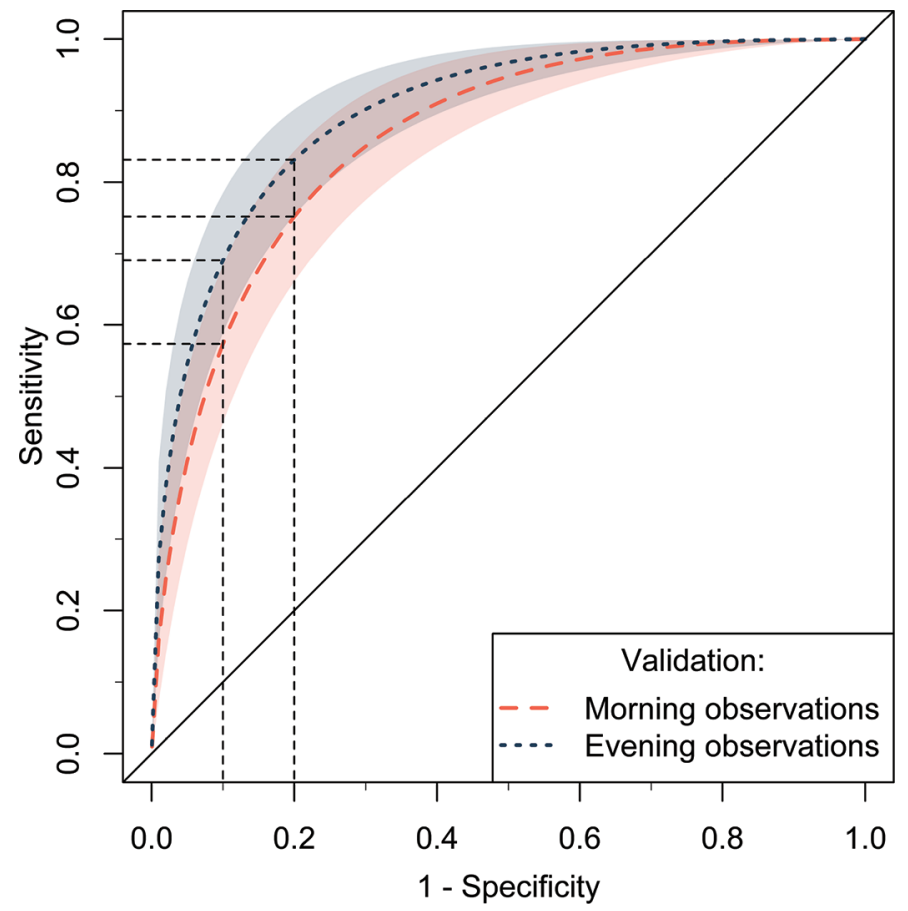

Figure 4. Smoothed receiver operating characteristic (ROC) curves for the identification of detrimental blood plasma nonesterified fatty acid (NEFA) concentrations $(\geq 0.6 \mathrm{mmol} / \mathrm{L})$. The NEFA concentrations were predicted with partial least squares regression models trained on morning and evening observations (full model) of the calibration set. The mean values (lines) and 95\% CI (areas) for the smoothed ROC curves are provided for morning (red, dashed) and evening observations (blue, dotted) of the validation set.

spectra of evening milk samples compared with those based on the paired morning milk samples, especially for cows with detrimental blood NEFA concentrations (Figure $3 \mathrm{~b}$ ). It might be interesting in future research to study these dynamics in more detail by measuring the blood NEFA level at frequent intervals and investigating the link with the MIR spectra of morning and evening milk samples on that day and the days that follow. Hence, considering a detailed image of the blood NEFA concentrations over the entire period in which the respective milk was produced would likely result in a stronger relation with relevant milk biomarkers.

The fact that the morning blood NEFA concentration can be predicted more accurately when the full model is applied on MIR spectra of evening milk rather than morning milk suggests that the evening milk samples contain more information on the morning blood NEFA concentration or that the morning milk samples are more subject to interfering effects. Nevertheless, training the model solely on evening milk MIR spectra (evening model) did not improve prediction performance compared with the full model, not 
even when exclusively MIR spectra of evening milk samples are considered in the validation. Moreover, the performance of the evening model was worse when applied on MIR spectra of morning milk samples. This suggests that including morning milk MIR spectra in the calibration set makes the prediction model more robust for potential interfering parameters that vary independently of the cow's blood NEFA level. One of these interfering effects might be the total fat content in the milk, which is generally higher in evening milk than in morning milk (Forsbäck et al., 2010). Artificially doubling the number of blood NEFA records and linking them to the morning and evening milk MIR spectra, respectively, thus helps the prediction model to find an "average" response. Aside from the results obtained in our study, the full model is probably the most robust under practical conditions, where farms have varying milking and feeding frequencies compared with the morning and evening models created here.

\section{Prediction of Blood Plasma NEFA Concentration}

The regression coefficients in Figure 2 show that the PLSR models primarily use information from the fatrelated MIR absorption bands. During negative energy status, excessive amounts of NEFA are mobilized from the adipose tissue and part of them is transferred to the milk. These NEFA are particularly rich in longchain FA, such as C18:1 FA (Jorjong et al., 2014). Dórea et al. (2017) found a nonlinear relation $\left(\mathrm{R}^{2}=\right.$ $0.42, P<0.001)$ between the concentrations of NEFA in blood plasma and C18:1 FA in milk fat. Moreover, milk C18:1 FA increased nearly linearly with increasing blood NEFA for blood NEFA levels below $400 \mu \mathrm{Eq} / \mathrm{L}$, whereas milk C18:1 FA concentration was practically constant for blood NEFA concentrations above 800 $\mu \mathrm{Eq} / \mathrm{L}$. This suggests that the C18:1 FA concentration in milk fat saturates when the blood NEFA increases above a certain concentration. In contrast, Jorjong et al. (2014) suggested a linear relation $\left(\mathrm{R}^{2}=0.383\right)$ between the concentrations of NEFA in the blood plasma and $\mathrm{C} 18: 1$ cis-9 FA in the milk fat. However, their linear function slightly underestimated the milk C18:1 cis-9 FA for blood NEFA concentrations between 0.2 and $0.4 \mathrm{mmol} / \mathrm{L}$, whereas it overestimated milk C18:1 cis-9 FA for blood NEFA levels $<0.1$ and $>0.9 \mathrm{mmol} / \mathrm{L}$. Accordingly, the data of Jorjong et al. (2014) confirm the nonlinear trend found by Dórea et al. (2017). In our study, the predicted blood NEFA concentrations versus the actual blood NEFA levels (Figure 3b) followed a very similar nonlinear trend to milk $\mathrm{C} 18: 1 \mathrm{FA}$ in the studies of Dórea et al. (2017) and Jorjong et al. (2014). Therefore, it is likely that our PLSR models largely rely on the MIR absorption by $\mathrm{C} 18: 1$ and related FA in milk. Several researchers have explored MIR spectroscopy to predict the concentration of certain $\mathrm{FA}$ in milk, obtaining $\mathrm{R}^{2}$ values for the prediction of $\mathrm{C} 18: 1$ FA between 0.11 and 0.96 (Rutten et al., 2009; Afseth et al., 2010; Soyeurt et al., 2011). Mäntysaari et al. (2019) used the PLSR models developed by Soyeurt et al. (2011) to predict milk FA concentrations from milk MIR spectra and accordingly studied the relation between the predicted milk FA and blood NEFA concentration. It was found that $\mathrm{C} 18: 1$ cis-9 and the sum of C18:1 FA in milk had the highest correlation ( $\mathrm{r}=$ 0.73) with blood NEFA, confirming our hypothesis.

The predicted versus measured scatterplot in Figure $3 \mathrm{~b}$, as well as the RMSEP values in Table 4, show that the accuracy of the prediction of the blood NEFA from milk MIR spectra was limited, especially if the blood NEFA concentration is high. The full model resulted in RMSEP values of $0.197,0.182$, and $0.190 \mathrm{mmol} / \mathrm{L}$ when evaluated on morning observations, evening observations, or a mixed set of morning and evening observations of the validation set, respectively. Considering the standard deviations of the blood NEFA concentration for the different sets (Table 3 ), the $\mathrm{R}^{2}$ values were 0.502 , 0.613 , and 0.558 , respectively. Nevertheless, because of heteroscedasticity, the RMSEP and $\mathrm{R}^{2}$ values strongly depend on the proportion of observations with a high blood NEFA concentration in the respective data sets. To account for this nonlinear effect, we also explored a logarithmic transformation of the blood NEFA levels before applying PLSR, and nonlinear models such as convolutional neural networks, unfortunately without any success. Moreover, using a more balanced calibration set with a similar number of observations with high and low blood NEFA levels through bootstrapping did not improve the performance of the prediction model either (results not shown). Because of the heteroscedasticity of the prediction error, benchmarking our results against earlier studies is challenging and should be done with caution.

Dórea et al. (2017) obtained RMSE values of 169 to $220 \mu \mathrm{Eq} / \mathrm{L}$ (equivalent to $\mu \mathrm{mol} / \mathrm{L}$ ) and $\mathrm{R}^{2}$ values of 0.080 to 0.457 for the prediction of blood NEFA levels for individual cows from different linear combinations or ratios of milk FA concentrations obtained from GLC analysis. As the descriptive statistics for the blood NEFA are very similar between their data set and ours (Table 3), it is fair to compare the results of these 2 studies. The prediction errors reported by Dórea et al. (2017) are very close to those obtained in our study. Nevertheless, the performance of their models is reported only for the calibration set and thus might be overoptimistic. Additionally, the approach followed by Dórea et al. (2017) requires labor and cost-intensive FA isolation and GLC analysis. 
Mäntysaari et al. (2019) used a linear combination of $\mathrm{C} 18: 1$ cis-9 and medium-chain FA concentrations in milk, derived from evening milk MIR spectra, and lactation stage to predict the morning blood NEFA concentration, obtaining an $\mathrm{R}^{2}$ of 0.61 and an RMSECV of $0.182 \mathrm{mmol} / \mathrm{L}$. A similar approach in the same study using morning milk MIR spectra resulted in an $\mathrm{R}^{2}$ of 0.52 and an RMSECV of $0.198 \mathrm{mmol} / \mathrm{L}$. Although these results only represent the cross-validation of the model, and thus might be overoptimistic, they are in close agreement with results obtained for the independent validation in our study.

Recently, Benedet et al. (2019), Grelet et al. (2019), and Luke et al. (2019) published PLSR models that predict blood NEFA levels directly from the MIR spectra of raw milk samples. The prediction performance of Grelet's model $\left(\mathrm{R}^{2}=0.39\right.$, RMSECV $\left.=344 \mu \mathrm{Eq} / \mathrm{L}\right)$ is based only on cross-validation of the calibration set and should thus be confirmed on an external validation set (Grelet et al., 2019). Still, their results are inferior to those obtained in our study because of the higher prediction error in the low blood NEFA range in their model. Moreover, although our full model is relatively accurate (RMSEP $\leq 0.143 \mathrm{mmol} / \mathrm{L}$ ) in this range, Grelet's model generally overestimates the low blood NEFA concentrations. In the high blood NEFA range, Grelet's model performed similarly to our models, both underestimating blood NEFA concentration. As a result, the prediction error of Grelet's model is nearly homoscedastic but worse than in our model, especially in the low blood NEFA range. Benedet et al. (2019) obtained a PLSR model that performed better than Grelet's model, with an $\mathrm{R}^{2}$ of 0.52 and a standard error of prediction $\left[S E P=\sqrt{n \times\left(R M S E P^{2}-\text { bias }^{2}\right) /(n-1)}\right]$ of 0.24 $\mathrm{mmol} / \mathrm{L}$ for a randomly selected validation set. Still, their results were slightly worse than those obtained in our study. In contrast to our models, Benedet's model uses only wavenumbers between 1,450 and $1,000 \mathrm{~cm}^{-1}$, ignoring the fat absorption bands at around 2,928, 2,858 , and $1,745 \mathrm{~cm}^{-1}$. Additionally, it should be noted that model performance typically deteriorates when it is applied on a completely independent herd, as illustrated by Luke et al. (2019). Moreover, the $\mathrm{R}^{2}$ of Luke's model decreased from 0.61 for a randomly selected validation set, covering the same herds as the calibration set, to 0.45 for a totally independent herd. The better performance of our full model was likely the result of a higher number of calibration samples ( $\mathrm{n}=$ 790) combined with well-controlled timing and protocol for blood and milk sample collection (Quiroz-Rocha et al., 2010).
In the current study, only milk MIR spectra were considered as inputs to predict the respective blood NEFA concentrations. Nevertheless, the relationship between concentrations of milk biomarkers and blood NEFA might change with parity, lactation stage, daily milk yield, breed, and season. Therefore, accounting for this information in the prediction model could further improve its performance. Mäntysaari et al. (2019) obtained a slight improvement of their model that predicts the blood NEFA from milk FA concentrations by also including information on lactation stage. In that study, a simple linear approach without any interactions was used, whereas the actual relationship might be much more complex. Therefore, future research should focus on how the relation between blood NEFA and milk metabolites is influenced by these factors and how this knowledge could help improve the accuracy and robustness of the prediction models.

\section{ROC Analysis}

Unlike the regression analysis, in which the RMSE is subject to the effect of heteroscedasticity, the ROC analysis is less dependent on the relative number of observations with a high blood NEFA concentration. Accordingly, these ROC analyses allow for a more objective comparison among different studies. Dórea et al. (2017) obtained their best results to identify cows with detrimental blood NEFA concentrations $(\geq 600 \mu \mathrm{Eq} / \mathrm{L})$ based on milk C13:0 FA using a threshold of 0.036 $\mathrm{g}$ of FA/100 $\mathrm{g}$ of milk fat. This resulted in an AUC, sensitivity, and specificity of $0.90,0.859$, and 0.823 , respectively. The ROC curve to detect detrimental blood NEFA levels based on milk C18:1 cis-9 FA reported by Jorjong et al. (2014) had a sensitivity of 0.75 and 0.5 at a specificity of 0.79 and 0.935 , respectively. The full model obtained in our approach and applied on the evening observations resulted in an AUC of 0.898 and a sensitivity of 0.831 at a specificity of 0.8 . The same model applied on morning observations had an AUC of 0.860 and a sensitivity of 0.752 at a specificity of 0.8 (Appendix Table A2). Therefore, we concluded that our model, which only requires MIR spectral analysis of raw milk, is not inferior to more complex techniques that require characterization of certain FA in the milk fat. A similar approach followed by Luke et al. (2019) to identify elevated blood NEFA levels resulted in AUC values of 0.87 and 0.82 , sensitivities of 0.73 and 0.25 , and specificities of 0.81 and 0.90 for a randomly selected and a completely independent validation set, respectively. Thus, our model tended to be slightly more robust than that of Luke et al. (2019). 
The validation on 3 independent herds suggests that the model can be used on other herds too. Nevertheless, this remains to be evaluated and confirmed. Although the prediction accuracy was far from excellent, the developed model is indicative of the blood NEFA concentration and thus might provide valuable information to further improve genetics, nutrition, and management of dairy cows. Because it can be applied on millions of historical and future milk MIR spectra, this approach could reveal information on the energy status of individual cows, herds, and pedigrees. This could result in improved estimations of breeding values and the identification of specific genetic markers for metabolic resilience. For practical use of the prediction model, however, the model will need to be compatible with 50/50 mixtures of milk from the morning and evening milking sessions of individual cows. This could influence the prediction performance of the model and should be further investigated.

\section{CONCLUSIONS}

In this study, we aimed to predict blood NEFA concentrations in individual dairy cows from their milk MIR spectra. The best model was obtained after training on MIR spectra of both morning and evening milk samples. The NEFA concentrations of blood plasma samples taken in the morning were predicted with a slightly higher accuracy if the model was applied on MIR spectra of evening milk samples. The obtained prediction accuracy was acceptable for low blood NEFA levels but unsatisfactory if the blood NEFA concentration was high. Nevertheless, low versus intermediate or high blood NEFA levels could be discriminated to identify 83 out of 100 cows with detrimental blood NEFA levels; only 20 out of 100 healthy cows were wrongly classified. This opens opportunities for identifying cows at risk of negative energy status and studying the metabolic resilience of individual cows and pedigrees.

\section{ACKNOWLEDGMENTS}

This study was funded by the Finnish Ministry of Agriculture and Forestry (Makera; 1844/312/2012; Helsinki, Finland), Valio Ltd. (Helsinki, Finland), Faba Co-op (Vantaa, Finland), Viking Genetics (Hollola, Finland), Finnish Cattle Breeding Foundation (Helsinki, Finland), and Raisioagro Ltd. (Raisio, Finland). Ben Aernouts and Ines Adriaens were funded as postdoctoral fellow (11ZG916N) and aspirant fellow (12K3916N), respectively, by Research Foundation Flanders (FWO, Brussels, Belgium). Ben Aernouts obtained additional funding from Research Foundation Flanders to perform a long research stay abroad at the Natural Resources Institute of Finland (Luke; grant V407018N). The authors have not stated any conflicts of interest.

\section{REFERENCES}

Adewuyi, A. A., E. Gruys, and F. J. C. M. V. Eerdenburg. 2005. Non esterified fatty acids (NEFA) in dairy cattle. A review. Vet. Q. 27:117-126. https://doi.org/10.1080/01652176.2005.9695192.

Aernouts, B., E. Polshin, W. Saeys, and J. Lammertyn. 2011. Mid-infrared spectrometry of milk for dairy metabolomics: a comparison of two sampling techniques and effect of homogenization. Anal. Chim. Acta 705:88-97. https://doi.org/10.1016/j.aca.2011.04.018.

Afseth, N. K., H. Martens, Å. Randby, L. Gidskehaug, B. Narum, K. Jørgensen, S. Lien, and A. Kohler. 2010. Predicting the fatty acid composition of milk: A comparison of two Fourier transform infrared sampling techniques. Appl. Spectrosc. 64:700-707. https://doi .org/10.1366/000370210791666200.

Barnes, R. J., M. S. Dhanoa, and S. J. Lister. 1989. Standard normal variate transformation and de-trending of near-infrared diffuse reflectance spectra. Appl. Spectrosc. 43:772-777. https://doi.org/10 $.1366 / 0003702894202201$.

Beer. 1852. Bestimmung der Absorption des rothen Lichts in farbigen Flüssigkeiten. Ann. Phys. 162:78-88. https://doi.org/10.1002/ andp. 18521620505 .

Benedet, A., M. Franzoi, M. Penasa, E. Pellattiero, and M. De Marchi. 2019. Prediction of blood metabolites from milk mid-infrared spectra in early-lactation cows. J. Dairy Sci. 102:11298-11307. https:// doi.org/10.3168/jds.2019-16937.

Bjerre-Harpøth, V., N. C. Friggens, V. M. Thorup, T. Larsen, B. M. Damgaard, K. L. Ingvartsen, and K. M. Moyes. 2012. Metabolic and production profiles of dairy cows in response to decreased nutrient density to increase physiological imbalance at different stages of lactation. J. Dairy Sci. 95:2362-2380. https://doi.org/10 $.3168 /$ jds.2011-4419.

Blum, J. W., R. M. Bruckmaier, P. Y. Vacher, A. Münger, and F. Jans. 2000. Twenty-four-hour patterns of hormones and metabolites in week 9 and 19 of lactation in high-yielding dairy cows fed triglycerides and free fatty acids. J. Vet. Med. A Physiol. Pathol. Clin. Med. 47:43-60. https://doi.org/10.1046/j.1439-0442.2000 $.00266 . x$.

Bro, R., K. Kjeldahl, A. K. Smilde, and H. A. L. Kiers. 2008. Crossvalidation of component models: A critical look at current methods. Anal. Bioanal. Chem. 390:1241-1251. https://doi.org/10 .1007/s00216-007-1790-1.

Bro, R., and A. K. Smilde. 2014. Principal component analysis. Anal. Methods 6:2812-2831. https://doi.org/10.1039/C3AY41907J.

Cederkvist, H. R., A. H. Aastveit, and T. Næs. 2005. A comparison of methods for testing differences in predictive ability. J. Chemometr. 19:500-509. https://doi.org/10.1002/cem.956.

Chong, I. G., and C. H. Jun. 2005. Performance of some variable selection methods when multicollinearity is present. Chemom. Intell. Lab. Syst. 78:103-112. https://doi.org/10.1016/j.chemolab.2004 .12.011.

De Marchi, M., V. Toffanin, M. Cassandro, and M. Penasa. 2014. Invited review: Mid-infrared spectroscopy as phenotyping tool for milk traits. J. Dairy Sci. 97:1171-1186. https://doi.org/10.3168/ jds.2013-6799.

Dórea, J. R. R., E. A. French, and L. E. Armentano. 2017. Use of milk fatty acids to estimate plasma nonesterified fatty acid concentrations as an indicator of animal energy balance. J. Dairy Sci. 100:6164-6176. https://doi.org/10.3168/jds.2016-12466.

Enjalbert, F., M. C. Nicot, C. Bayourthe, and R. Moncoulon. 2010. Ketone bodies in milk and blood of dairy cows: Relationship between concentrations and utilization for detection of subclinical ketosis. J. Dairy Sci. 84:583-589. https://doi.org/10.3168/jds.S0022 -0302(01)74511-0.

Fawcett, T. 2006. An Introduction to ROC analysis. Pattern Recognit. Lett. 27:861-874. https://doi.org/10.1016/j.patrec.2005.10.010. 
Forsbäck, L., H. Lindmark-Månsson, A. Andrén, M. Akerstedt, L. Andrée, and K. Svennersten-Sjaunja. 2010. Day-to-day variation in milk yield and milk composition at the udder-quarter level. J. Dairy Sci. 93:3569-3577. https://doi.org/10.3168/jds.2009-3015.

Franzoi, M., G. Niero, G. Visentin, M. Penasa, M. Cassandro, and M. De Marchi. 2019. Variation of detailed protein composition of cow milk predicted from a large database of mid-infrared spectra. Animals (Basel) 9:176. https://doi.org/10.3390/ani9040176.

Geladi, P., D. MacDougall, and H. Martens. 1985. Linearization and scatter-correction for near-infrared reflectance spectra of meat. Appl. Spectrosc. 39:491-500. https://doi.org/10.1366/ 0003702854248656.

Grelet, C., C. Bastin, M. Gelé, J.-B. Davière, M. Johan, A. Werner, R. Reding, J. A. Fernandez Pierna, F. G. Colinet, P. Dardenne, N. Gengler, H. Soyeurt, and F. Dehareng. 2016. Development of Fourier transform mid-infrared calibrations to predict acetone, $\beta$-hydroxybutyrate, and citrate contents in bovine milk through a European dairy network. J. Dairy Sci. 99:4816-4825. https://doi .org/10.3168/jds.2015-10477.

Grelet, C., J. A. Fernández Pierna, P. Dardenne, V. Baeten, and F. Dehareng. 2015. Standardization of milk mid-infrared spectra from a European dairy network. J. Dairy Sci. 98:2150-2160. https://doi .org/10.3168/jds.2014-8764.

Grelet, C., A. Vanlierde, M. Hostens, L. Foldager, M. Salavati, K. L. Ingvartsen, M. Crowe, M. T. Sorensen, E. Froidmont, C. P. Ferris, C. Marchitelli, F. Becker, T. Larsen, F. Carter, and F. Dehareng. 2019. Potential of milk mid-IR spectra to predict metabolic status of cows through blood components and an innovative clustering approach. Animal 13:649-658. https://doi.org/10.1017/ S1751731118001751.

ICAR. 2019. Section 12 - Guidelines for Milk Analysis. International Committee on Animal Recording, Rome, Italy.

ISO/IDF. 2013. Milk and liquid milk products-Guidelines for the application of mid-infrared spectrometry. Page 14 in International Standard ISO 9622:2013/IDF 141:2013. International Organization for Standardization, Geneva, Switzerland/ International Dairy Federation, Brussels, Belgium.

Jorjong, S., A. T. M. van Knegsel, J. Verwaeren, M. V. Lahoz, R. M. Bruckmaier, B. De Baets, B. Kemp, and V. Fievez. 2014. Milk fatty acids as possible biomarkers to early diagnose elevated concentrations of blood plasma nonesterified fatty acids in dairy cows. J. Dairy Sci. 97:7054-7064. https://doi.org/10.3168/jds.2014-8039.

Kemps, B. J., W. Saeys, K. Mertens, P. Darius, J. G. De Baerdemaeker, and B. De Ketelaere. 2010. The importance of choosing the right validation strategy in inverse modelling. J. Near Infrared Spectrosc. 18:231-237. https://doi.org/10.1255/jnirs.882.

LeBlanc, S. 2010. Monitoring metabolic health of dairy cattle in the transition period. J. Reprod. Dev. 56(S):S29-S35. https://doi.org/ 10.1262/jrd.1056s29.

Leroy, J. L. M. R., P. Bossaert, G. Opsomer, and P. E. J. Bols. 2011. The effect of animal handling procedures on the blood non-esterified fatty acid and glucose concentrations of lactating dairy cows. Vet. J. 187:81-84. https://doi.org/10.1016/j.tvjl.2009.10.003.

Leroy, J. L. M. R., T. Vanholder, B. Mateusen, A. Christophe, G. Opsomer, A. de Kruif, G. Genicot, and A. Van Soom. 2005. Nonesterified fatty acids in follicular fluid of dairy cows and their effect on developmental capacity of bovine oocytes in vitro. Reproduction 130:485-495. https://doi.org/10.1530/rep.1.00735.

Luke, T. D., S. Rochfort, W. J. Wales, V. Bonfatti, L. Marett, and J. E. Pryce. 2019. Metabolic profiling of early-lactation dairy cows using milk mid-infrared spectra. J. Dairy Sci. 102:1747-1760. https://doi.org/10.3168/jds.2018-15103.

Mäntysaari, P., E. A. Mäntysaari, T. Kokkonen, T. Mehtiö, S. Kajava, C. Grelet, P. Lidauer, and M. H. Lidauer. 2019. Body and milk traits as indicators of dairy cow energy status in early lactation. J. Dairy Sci. 102:7904-7916. https://doi.org/10.3168/jds.2018-15792.

Martens, H., and T. Næs. 1987. Multivariate calibration by data compression. P. C. Williams and K. Norris, ed. American Association of Cereal Chemists, St Paul, MN.

Meier, S., E. S. Kolver, G. A. Verkerk, and J. R. Roche. 2010. Effects of divergent Holstein-Friesian strain and diet on diurnal patterns of plasma metabolites and hormones. J. Dairy Res. 77:432-437. https://doi.org/10.1017/S002202991000052X.

Nørgaard, L., A. Saudland, J. Wagner, J. P. Nielsen, L. Munck, and S. B. Engelsen. 2000. Interval partial least-squares regression (iPLS): A comparative chemometric study with an example from near-infrared spectroscopy. Appl. Spectrosc. 54:413-419. https://doi.org/ 10.1366/0003702001949500.

Ospina, P. A., D. V. Nydam, T. Stokol, and T. R. Overton. 2010a. Associations of elevated nonesterified fatty acids and $\beta$-hydroxybutyrate concentrations with early lactation reproductive performance and milk production in transition dairy cattle in the northeastern United States. J. Dairy Sci. 93:1596-1603. https: //doi.org/10.3168/jds.2009-2852.

Ospina, P. A., D. V. Nydam, T. Stokol, and T. R. Overton. 2010b. Evaluation of nonesterified fatty acids and $\beta$-hydroxybutyrate in transition dairy cattle in the northeastern United States: Critical thresholds for prediction of clinical diseases. J. Dairy Sci. 93:546554. https://doi.org/10.3168/jds.2009-2277.

Quiroz-Rocha, G. F., S. J. LeBlanc, T. F. Duffield, B. Jefferson, D. Wood, K. E. Leslie, and R. M. Jacobs. 2010. Short communication: Effect of sampling time relative to the first daily feeding on interpretation of serum fatty acid and $\beta$-hydroxybutyrate concentrations in dairy cattle. J. Dairy Sci. 93:2030-2033. https://doi .org/10.3168/jds.2009-2141.

R Core Team. 2017. R: A language and environment for statistical computing. R Core Team, Vienna, Austria.

Robin, X., N. Turck, A. Hainard, N. Tiberti, F. Lisacek, J. Sanchez, and M. Müller. 2011. pROC: An open-source package for R and $\mathrm{S}+$ to analyze and compare ROC curves. BMC Bioinformatics 12:77. https://doi.org/10.1186/1471-2105-12-77.

Ruckstuhl, A. F., M. P. Jacobson, R. W. Field, and J. A. Dodd. 2001. Baseline subtraction using robust local regression estimation. J. Quant. Spectrosc. Radiat. Transf. 68:179-193. https://doi.org/10 .1016/S0022-4073(00)00021-2.

Rutten, M. J. M., H. Bovenhuis, K. A. Hettinga, H. J. F. van Valenberg, and J. A. M. van Arendonk. 2009. Predicting bovine milk fat composition using infrared spectroscopy based on milk samples collected in winter and summer. J. Dairy Sci. 92:6202-6209. https: //doi.org/10.3168/jds.2009-2456.

Salin, S., J. Taponen, K. Elo, I. Simpura, A. Vanhatalo, R. Boston, and T. Kokkonen. 2012. Effects of abomasal infusion of tallow or camelina oil on responses to glucose and insulin in dairy cows during late pregnancy. J. Dairy Sci. 95:3812-3825. https://doi.org/10 $.3168 /$ jds.2011-5206.

Savitzky, A., and M. J. E. Golay. 1964. Smoothing and differentiation of data by simplified least squares procedures. Anal. Chem. 36:1627-1639. https://doi.org/10.1021/ac60214a047.

Scalia, D., N. Lacetera, U. Bernabucci, K. Demeyere, L. Duchateau, and C. Burvenich. 2006. In vitro effects of nonesterified fatty acids on bovine neutrophils oxidative burst and viability. J. Dairy Sci. 89:147-154. https://doi.org/10.3168/jds.S0022-0302(06)72078-1.

Snee, R. 1977. Validation of regression models: Methods and examples. Technometrics 19:415-428. https://doi.org/10.1080/00401706 .1977 .10489581$.

Soyeurt, H., D. Bruwier, J.-M. Romnee, N. Gengler, C. Bertozzi, D. Veselko, and P. Dardenne. 2009. Potential estimation of major mineral contents in cow milk using mid-infrared spectrometry. J. Dairy Sci. 92:2444-2454. https://doi.org/10.3168/jds.2008-1734.

Soyeurt, H., F. Dehareng, N. Gengler, S. Mcparland, E. Wall, D. P. Berry, M. Coffey, and P. Dardenne. 2011. Mid-infrared prediction of bovine milk fatty acids across multiple breeds, production systems, and countries. J. Dairy Sci. 94:1657-1667. https://doi.org/ 10.3168/jds.2010-3408.

Van Haelst, Y. N. T., A. Beeckman, A. T. M. Van Knegsel, and V. Fievez. 2008. Short communication: Elevated concentrations of oleic acid and long-chain fatty acids in milk fat of multiparous subclinical ketotic cows. J. Dairy Sci. 91:4683-4686. https://doi .org/10.3168/jds.2008-1375.

Westad, F., and H. Martens. 2000. Variable selection in near infrared spectroscopy based on significance testing in partial least squares 
regression. J. Near Infrared Spectrosc. 8:117-124. https://doi.org/ 10.1255 /jnirs. 271

Williams, P., and K. Norris. 2001. Near-Infrared Technology in the Agricultural and Food Industries. 2nd ed. American Association of Cereal Chemists, St. Paul, MN.

\section{ORCIDS}

Ben Aernouts (ํ) https://orcid.org/0000-0001-6266-3019

Ines Adriaens $\odot$ https://orcid.org/0000-0001-9768-2308
José Diaz-Olivares @ https://orcid.org/0000-0001-6178-0088

Wouter Saeys (ํ) https://orcid.org/0000-0002-5849-4301

Päivi Mäntysaari @ https://orcid.org/0000-0002-3534-4802

Tuomo Kokkonen ๑ https://orcid.org/0000-0001-7176-1120

Terhi Mehtiö @ $\odot$ https://orcid.org/0000-0002-6401-882X

Sari Kajava @ https://orcid.org/0000-0002-1769-4622

Paula Lidauer @ ( https://orcid.org/0000-0001-7608-2629

Martin H. Lidauer (®) https://orcid.org/0000-0003-0508-9991

Matti Pastell ৫ https://orcid.org/0000-0002-5810-4801

\section{APPENDIX}

Table A1. Mean values of the sensitivities (Se) and the 95\% CI of the sensitivities and specificities (Sp) at given specificities for the morning and evening observations of the validation set

\begin{tabular}{|c|c|c|c|c|c|c|}
\hline \multirow[b]{2}{*}{ Sp } & \multicolumn{3}{|c|}{ Morning observations } & \multicolumn{3}{|c|}{ Evening observations } \\
\hline & $95 \%$ CI Sp & $\mathrm{Se}$ & $95 \%$ CI Se & $95 \%$ CI Sp & Se & $95 \%$ CI Se \\
\hline 0.700 & $0.600-0.794$ & 0.850 & $0.775-0.924$ & $0.584-0.800$ & 0.902 & $0.841-0.953$ \\
\hline 0.750 & $0.661-0.830$ & 0.807 & $0.725-0.890$ & $0.648-0.835$ & 0.872 & $0.803-0.932$ \\
\hline 0.800 & $0.724-0.866$ & 0.752 & $0.661-0.844$ & $0.714-0.870$ & 0.831 & $0.754-0.902$ \\
\hline 0.850 & $0.788-0.902$ & 0.678 & $0.577-0.778$ & $0.781-0.905$ & 0.775 & $0.687-0.856$ \\
\hline 0.900 & $0.852-0.939$ & 0.573 & $0.463-0.683$ & $0.848-0.941$ & 0.690 & $0.589-0.785$ \\
\hline 0.950 & $0.918-0.974$ & 0.410 & $0.293-0.533$ & $0.916-0.974$ & 0.545 & $0.425-0.660$ \\
\hline
\end{tabular}


a)

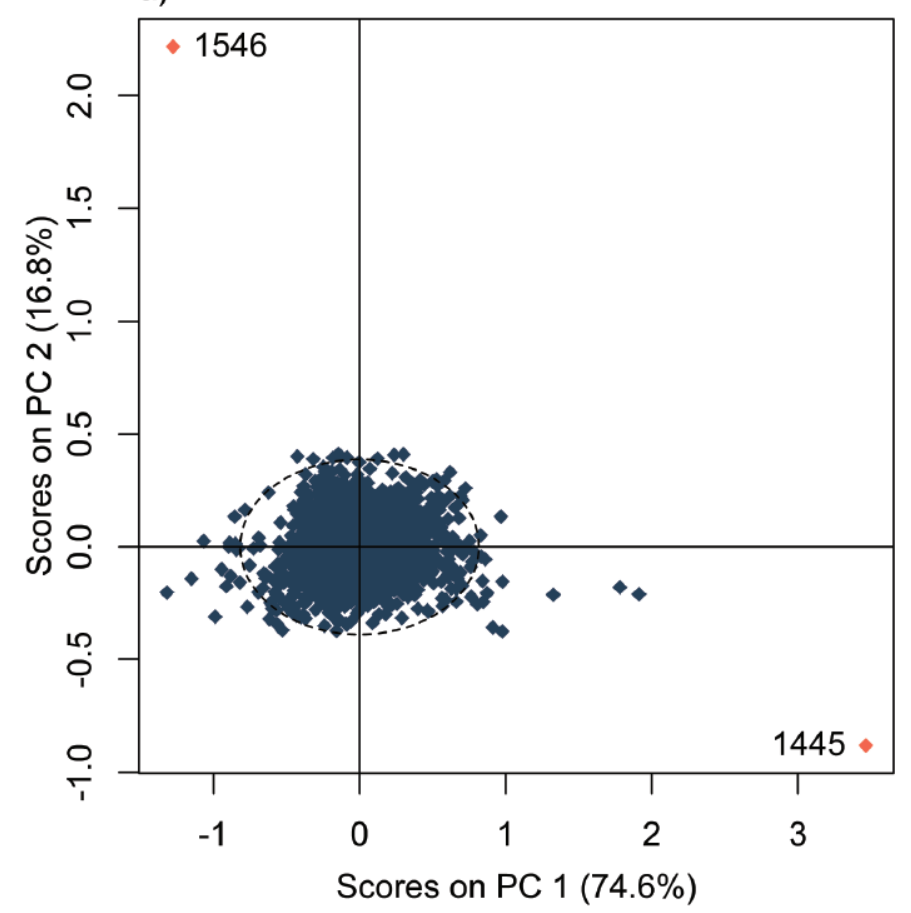

b)

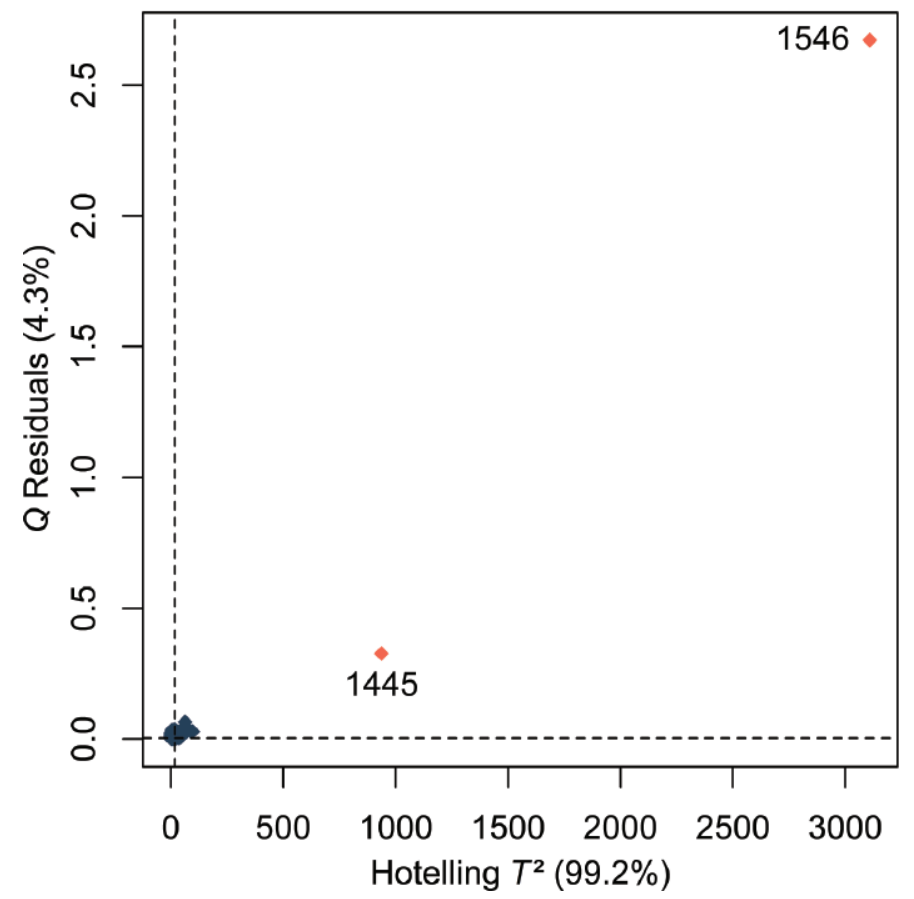

Figure A1. (a) Score plot of principal component (PC) 1 versus $\mathrm{PC} 2$ of the principal component analysis (PCA) on all 1,585 meancentered mid-infrared transmittance spectra. The dashed ellipse represents the $99 \%$ confidence limits of the scores on PC1 and PC2. (b) The influence plot ( $Q$ residual vs. Hotelling $T^{2}$ statistics) for the PCA model with 7 PC. The dashed lines represent the $99 \%$ confidence limits on the 2 statistics, respectively. In both figures, each dot represents a different sample spectrum and the red dots (with sample number) indicate potential outlier spectra. 